\title{
Effect of field-aligned beam on upstream wave excitation and particle scattering in the earth's foreshock: One-dimensional PIC simulation
}

\author{
F. Otsuka*, S. Matsukiyo, and T. Hada \\ Faculty of Engineering Sciences, Kyushu University \\ Kasuga-Koen 6-1, Kasuga, Fukuoka, Japan \\ E-mail: otsuka@esst.kyushu-u.ac.jp, matsukiy@esst.kyushu-u.ac.jp, \\ hada@esst.kyushu-u.ac.jp
}

\begin{abstract}
The Earth's foreshock extends to a large domain of upstream quasi-parallel bow shock, and is characterized by a presence of field-aligned beams (FABs), diffuse ions, ultra-low frequency (ULF) waves, high frequency whistler waves, shocklets, and so on. Kinetic self-consistent numerical simulation is one of the key tools to analyze detailed physics of the foreshock which has not been clearly understood. Because of the necessity of the large simulation domain, a full particle-in-cell (PIC) simulation of quasi-parallel shock has seldom been performed. In this paper we show preliminary results of a long-term and large-scale one-dimensional full PIC simulation of the quasi-parallel collisionless shock with the Alfvén Mach number 6.6 and shock angle 20 degrees. The FAB component is observed far upstream with the beam velocity of 10.5 times the Alfvén velocity and the beam density of $0.5 \%$ of the background plasma. This FAB generates right-handed Alfvén waves in the plasma rest frame via resonant mode instability, and the excited waves are amplified as approaching the shock during the plasma convection. The number densities of energetic particles for both electrons and ions also increase as approaching the shock.
\end{abstract}

35th International Cosmic Ray Conference

10-20 July, 2017

Bexco, Busan, Korea

* Speaker. 


\section{Introduction}

It is well-known that some of the solar wind ions are reflected at the quasi-perpendicular side of the earth's bow shock, and are streaming away from the shock along the upstream magnetic field. These field aligned beams (FABs) generate electromagnetic waves via beam-driven instabilities. Because of the combined geometry of the curved bow shock and the magnetic field direction, these excited waves travel into the quasi-parallel side of the shock and evolve nonlinearly during the solar wind convection. In addition, the particles reflected or accelerated at the quasi-parallel side of the bow shock can also be a source of the beam-driven instabilities in the foreshock. Balogh and Treumann (2013) review the physical processes in the earth's foreshock in detail [1]. A number of in situ observations exhibits a variety of foreshock waves such as monochromatic ULF wave, turbulent waves including from low to high frequency waves, and nonlinear waves such as short large amplitude magnetic structures (SLAMS) and shocklets. These waves scatter the energetic ions including the FABs themselves and the so-called diffuse ions. However, the mechanisms of the excitation and the evolution of the waves and subsequent diffusion and acceleration of energetic particles have not been well understood.

Kinetic self-consistent numerical simulation is one of the key tools to analyze detailed physics of the foreshock. There are a number of past studies of quasi-parallel collisionless shock using hybrid simulation, which treats ions as discrete particles and electrons as a massless fluid. Giacalone (2004) and Sugiyama (2011) found that both magnetic field strength and differential density of energetic ions upstream of the shock increase as approaching the shock, and that downstream energetic ion spectrum shows power-law nature [2,3]. These features are qualitatively consistent with the scenario of the diffusive shock acceleration (DSA) [4]. However, hybrid simulation ignores electron dynamics and cannot treat high frequency waves. Full particle-in-cell (PIC) simulation, in which both ions and electrons are treated as discrete particles, can deal with these problems.

The full PIC simulation of quasi-parallel shock is seldom performed. Scholer et al.(2003) and Tsubouchi and Lembége (2004) showed that the low-frequency upstream waves steepen and evolve into pulsations or SLAMS near the shock. They also discussed the ion phase space vortex, whistler wave dynamics, and the shock reformation $[5,6]$. In their full PIC simulations, the ratio of the electron plasma frequency $\left(\omega_{p e}\right)$ to the electron gyrofrequency $\left(\Omega_{e}\right)$ is relatively low, compared with a realistic parameter in the solar wind. Namely, $\omega_{p e} / \Omega_{e}=\sqrt{10}$ for Scholer et al. (2003) and $\omega_{p e} / \Omega_{e}=4$ for Tsubouchi \& Lembége (2004). On the other hand, $\omega_{p e} / \Omega_{e} \sim 10^{2}$ for a typical solar wind, where the plasma density $\sim 5 / c c$ and the ion gyrofrequency $\sim 0.1 \mathrm{~Hz}$ are assumed. Assuming these parameters, the aforementioned authors could simulate the well developed upstream field with reasonable CPU time. However, their discussions are limited to the vicinity of the shock, since their system sizes are 200 and 700 times the ion inertial length, for Scholer et al.(2003) and Tsubouchi and Lembége (2004), respectively. In order to investigate wave evolution and particle scattering in the extended foreshock for more realistic parameters, a larger simulation domain is needed. In this paper we perform one-dimensional full PIC simulation of a quasi-parallel shock to model the Earth's bow shock, using the spatial scales one-order larger than the past simulations. 

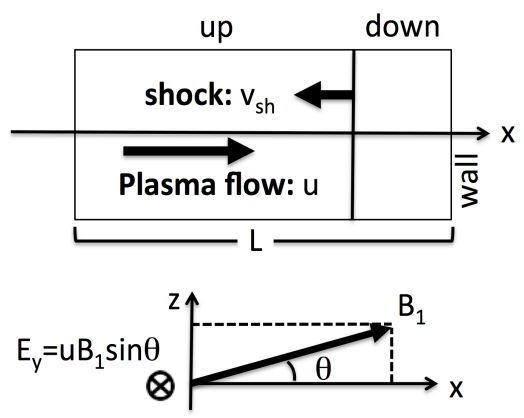

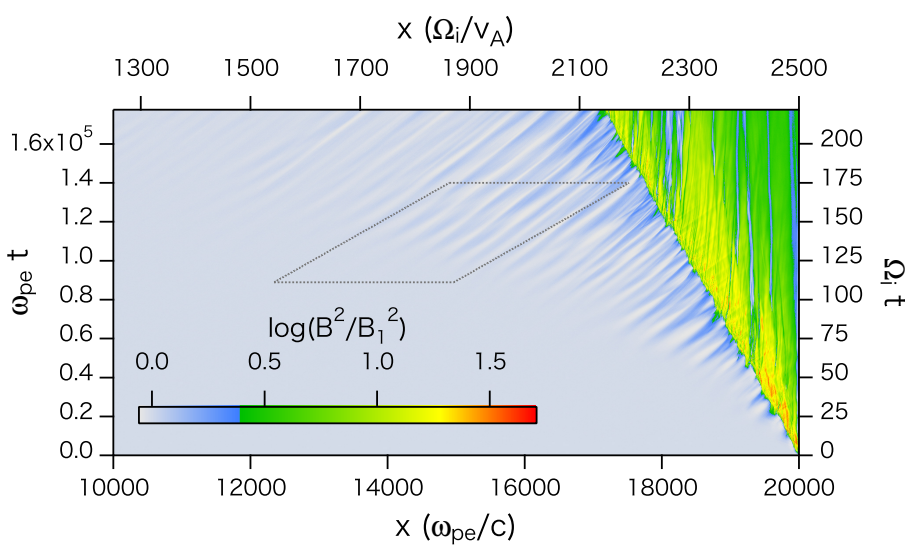

Figure 1: (Left) Simulation setup. (Right) Evolution of magnetic field intensity. The surrounded region by gray lines is the region co-moving with the plasma flow, and corresponds to the region in Fig.3(b).

\section{Method and Results}

Left panel of Figure 1 shows the simulation setup. The system has one-dimension along the $x$-axis, and ion-electron plasma flows from the left to right along the axis with a speed of $u$. Background upstream magnetic field $\left(B_{1}\right)$ lies in the $x-z$ plane with the shock angle, $\theta=20$ degrees. Then, motional electric field, $E_{y}=u B_{1} \sin \theta$, exists along the $y$ axis. At the left boundary a fresh plasma is injected continuously, and at the right boundary the plasma is specularly reflected at a rigid wall. Then, the incoming and reflected plasma flows are mixed near the wall boundary to form downstream plasma, and a shock is produced between the upstream and downstream media and propagates toward the negative $x$-axis. Hence, the simulation is in the downstream frame of reference. The plasma parameters are as follows. The incoming flow speed is $u=0.05 c$, where $c$ is the light speed. The ion and electron mass ratio is $m_{i} / m_{e}=64$, electron plasma frequency is $\omega_{p e}=12.5 \Omega_{e}$. The temperatures for ions and electrons are the same with $T_{e}=T_{i}=1.6 \times 10^{-3} m_{e} c^{2}=0.01 m_{i} u^{2}$. Accordingly, Alfvén velocity is $v_{A} / c=0.01$, plasma beta is 0.5 for both species. The system size is $L=2 \times 10^{4} c / \omega_{p e}=2500 v_{A} / \Omega_{i}$ and calculation time is $t \omega_{p e}=1.77 \times 10^{5}$, corresponding to $t \Omega_{i}=221.25$. The time step and spatial grid are $\omega_{p e} \Delta t=0.04$ and $\Delta x=0.04 \lambda_{D_{e}}$, respectively, where $\lambda_{D_{e}}=v_{t h, e} / \omega_{p e}$ is the Debye length. There are 128 macroparticles per cell for both species. Right panel of Figure 1 shows an image of the magnetic field intensity (color scale) in time (vertical axis) and space (bottom axis). Here, the magnetic field is $\mathbf{B}=\left(B_{1} \cos \theta, B_{y}(x, t), B_{1} \sin \theta+B_{z}(x, t)\right)$. Shock is propagating to the negative $x$ direction with shock speed, $v_{s h}=-0.016 c$; then Alfvén Mach number is 6.6. The stripes seen upstream indicates the so-called ULF waves convected by the solar wind. As seen from Fig.1, the ULF waves are excited upstream after $t \Omega_{i} \sim 50$ and are extended to a large spatial domain, $1500 \sim 2100 v_{A} / \Omega_{i}$, at the end of the simulation.

Figure 2 (a) and (b) show the magnetic field fluctuations ( $B_{y}$ component) and the number densities of energetic particles, respectively, at $\Omega_{i} t=175$ as a function of spatial position. The shock surface is located at $\sim 2220 v_{A} / \Omega_{i}$. In (b) energetic particles are selected with their kinetic energies higher than $0.13 m_{i} u^{2}$, and the thick and normal lines are for electrons and ions, respectively. The 


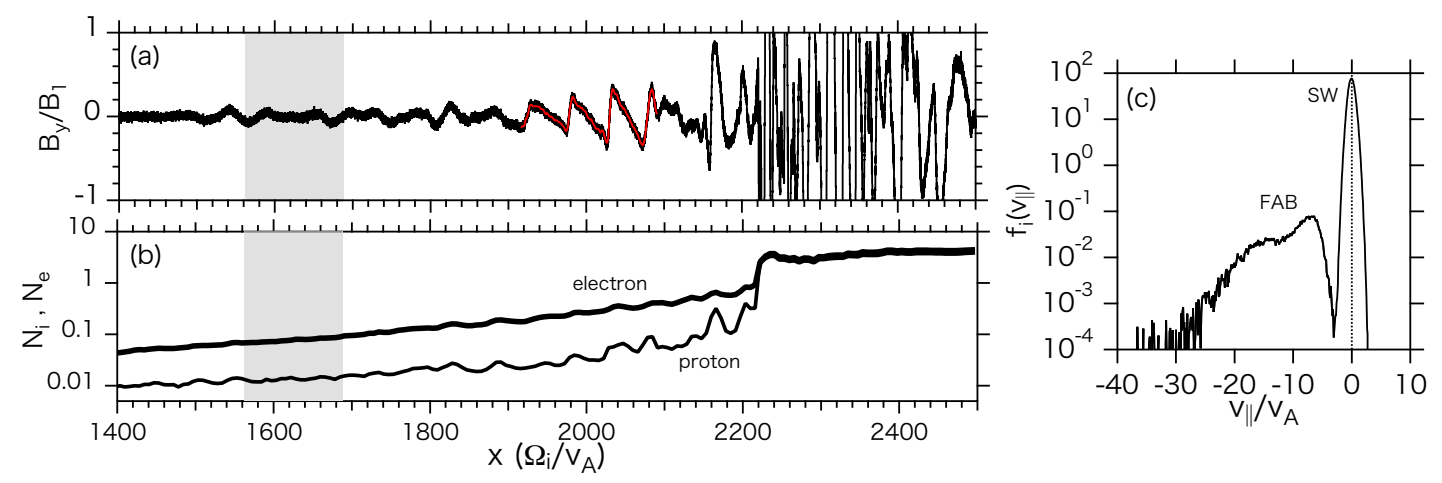

Figure 2: Results at $\Omega_{i} t=175$. (a) Magnetic field fluctuations ( $B_{y}$ component), (b) energetic particle densities, and (c) ion distribution as a function of the parallel velocity. In (a) the data shown by the red line corresponds to $B_{t 2}=B_{y}$ in Fig.3(a). In (b) the energetic particles are considered as their energies, measured in the upstream rest frame, larger than $0.13 m_{i} u^{2}$. In (c) the distribution is evaluated using the ions located in the gray region shown in (a) and (b).
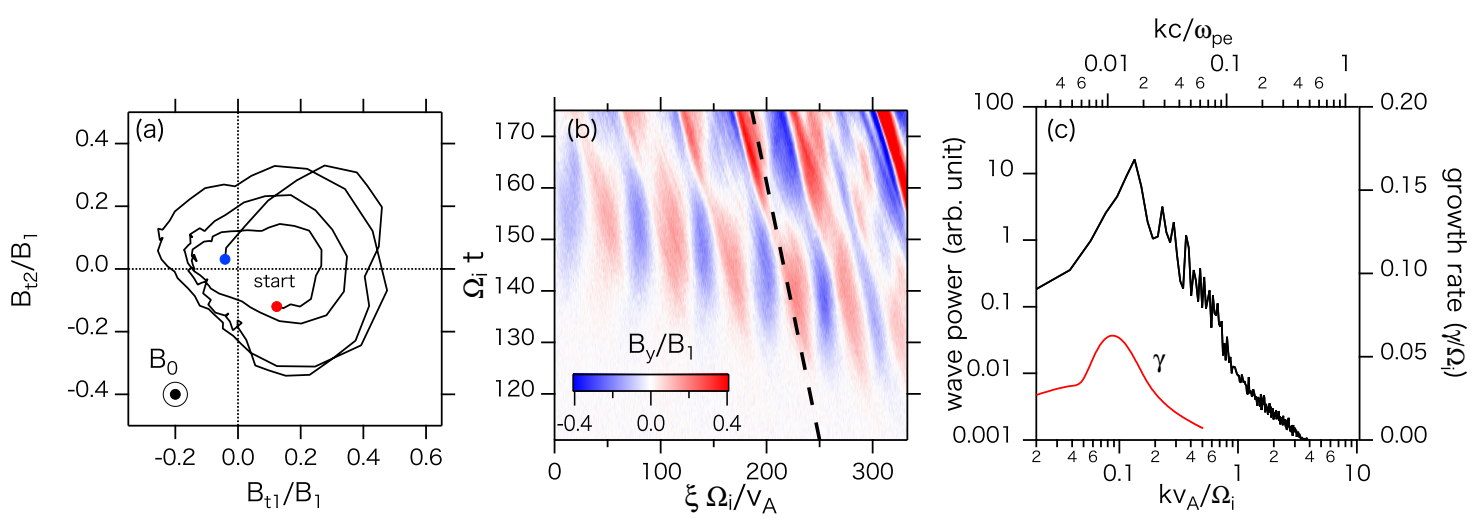

Figure 3: (a) Hodogram of the transverse magnetic field components, (b) image of the $B_{y}$ component in the upstream rest frame, (c) magnetic field wave power (shown by the black line with the left axis) and wave growth rate (shown by the red line with the right axis). In (b) the slope of the dashed line indicates Alfvén velocity. In (c) the FAB parameters obtained from Fig.2(c) are used for the evaluation of the growth rate.

wave amplitude as well as the number densities of both energetic particles are increased, as one approaches the shock. We emphasize here that the number of energetic electrons is one-order larger than that of energetic ions at elapsed time, $\Omega_{i} t=175$. Figure 2 (c) shows the ion distribution as a function of parallel velocity in the upstream rest frame. Here the distribution is evaluated using the ions far upstream, i.e. $1562.5<x \Omega_{i} / v_{A}<1687.5$ indicated by the gray region in Fig.2(a) and (b). The first intense peak at $v_{\|}=0$ represents the solar wind component, and the second peak indicates the FAB component. The FAB properties are summarized as follows. Relative beam density with respect to the total ion density is $0.515 \%$, and the beam velocity is $-10.5 v_{A}$. The thermal velocities of the FAB parallel and perpendicular to the upstream field are $7.3 v_{A}$ and $4.5 v_{A}$, respectively. If the solar wind ions are specularly reflected at the shock in the de-Hoffmann-Teller frame, the beam speed in the solar wind frame becomes $v_{b, H T}=2 M_{A} v_{A} / \cos \theta=14.0 v_{A}$. Hence, most of the 

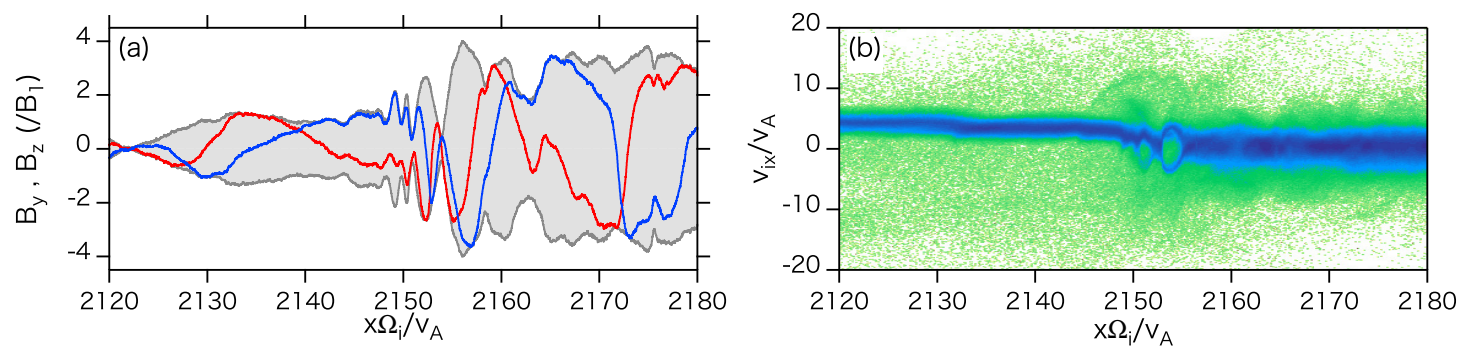

Figure 4: (a) Further developed $B_{y}$ (red) and $B_{z}$ (blue) components and (b) ion distribution in $v_{x}-x$ phase space at $\Omega_{i} t=219$. In (a) gray background indicates the envelope of the magnetic field.

FAB is approximately produced by the specular reflection of solar wind ions at the shock.

Let us discuss the excited wave properties in detail. Figure 3(a) shows the hodogram of magnetic field components transverse to the background field, $B_{t 1}=-B_{z} \cos \theta$ and $B_{t 2}=B_{y}$ to take along the $x$-axis at a fixed time $t \Omega_{i}=175$. The red and blue dots denote the starting and ending points. The corresponding spatial domain is shown as the red line in Fig.2(a). Almost circular helicity directed in anti-counterclockwise which we define positive wavenumber, is observed. Figure 3(b) shows spatio-temporal evolution of the upstream $B_{y}$ fluctuations in the upstream rest frame. Its original data is indicated as the region surrounded by the gray lines in the right panel of Fig.1. Here, $\xi$ represents the position in the solar wind rest frame, and is written as $\xi=x-x_{0}-u\left(t-t_{0}\right)$ where $x_{0}=1543 v_{A} / \Omega_{i}$ and $\Omega_{i} t_{0}=111$. The slope of dashed line in (b) denotes the Alfvén velocity. The waves are propagating away from the shock approximately with the Alfvén velocity. From (a) and (b) a typical frequency of the excited waves is identified as negative value, i.e., right-handed polarity. Figure 3(c) shows the wave power spectrum (shown by the black solid line in the left axis) and the wave growth rate, $\gamma$ (shown by the red line with the right axis), as functions of wavenumber, $k$. The wave power spectrum is obtained from Fourier transform in space and is averaged in time, using the data of $B_{y}$ (shown in (b)) and $B_{z}$ (not shown) components. The wave growth rate is numerically evaluated by Kyoto University Plasma Dispersion Analysis Package (KUPDAP) [7], using the FAB parameter described above. The spectrum is peaked at $k=0.13 \Omega_{i} / v_{A}$, and the growth rate is peaked at $k=0.063 \Omega_{i} / v_{A}$; thus the wavenumber of two peaks roughly coincide with each other. Therefore, the excited ULF waves are concluded to be right-handed Alfvén waves propagating away from the shock in the solar wind frame, and is driven by resonant mode instability, as Winske and Leroy (1984) has been explained it [8].

Figure 4(a) shows further developed $B_{y}$ (red) and $B_{z}$ (blue) components at $\Omega_{i} t=219$. It is seen that the whistler waves with frequency higher than the ULF is radiated in front of the shock ramp located at $\sim 2158 v_{A} / \Omega_{i}$. Figure 4(b) shows ion distribution in $v_{x}-x$ phase space at the same time of $\Omega_{i} t=219$. In the place where the whistler waves exist, some ions are trapped resulting in phase space vortices. This feature is well studied by Scholer et al.(2003) [5], and our result is qualitatively consistent with theirs.

\section{Summary}

We showed preliminary results of one-dimensional PIC simulation of quasi-parallel earth's 
bow shock, focusing on the effects of the FAB on the wave excitation and the evolution of the beam-wave system. We reproduced some essential features obtained in the past PIC simulation ([5]) and hybrid simulations ([2, 3]). Our obtained features are as follows: (1) generation of the $\mathrm{FAB}$, (2) right-handed Alfvén wave excitation through resonant mode instability, (3) increases in the number densities of the energetic ions and electrons, as well as the increase in wave intensity, as approaching the shock, (4) high-frequency whistler waves accompanied by ion vortices. Further investigation of particle scattering and acceleration induced by these waves will be reported soon.

\section{Acknowledgments}

This work was supported by Grant-in-Aid for Japan Society for the Promotion of Science (JSPS) Fellows (KAKENHI 15J40063) and JSPS Bilateral Joint Research Projects.

\section{References}

[1] A. Balogh, R.A. Treumann (2013), Physics of Collisionless Shocks, ISSI Scientific Report Series 12, Chapter 6, Springer New York Heidelberg Dordrecht London.

[2] J. Giacalone (2004), Large scale hybrid simulations of particle acceleration at a parallel shock, Astrophys. J., 609, 452-458.

[3] T. Sugiyama (2011), Time sequence of energetic particle spectra in quasiparallel shocks in large simulation systems Physics Plasma, 18, 022302.

[4] A.R. Bell. (1978), The acceleration of cosmic rays in shock fronts - I Mon. Not. R. astr. Soc., 182, $147-156$

[5] M. Scholer (2003), Short large-amplitude magnetic structures and whistler wave precursors in a full-particle quasi-parallel shock simulation J. Geophys. Res., 108, A7, 1273

[6] K. Tsubouchi and B. Lembége (2004), Full particle simulations of short large-amplitude magnetic structures (SLAMS) in quasi-parallel shocks J. Geophys. Res., 109, A02114

[7] H. Sugiyama et al. (2015), Electromagnetic ion cyclotron waves in the Earths magnetosphere with a kappa-Maxwellian particle distribution J. Geophys. Res., Space Physics, 120, 8426-8439.

[8] D. Winske and M.M. Leroy (1984), Diffuse ions produced by electromagnetic ion beam instabilities J. Geophys. Res., 85, A5, 2673-2688 